\title{
The empirical study of market share and marketing strategy based on Markov chain model
}

\author{
Tian-Yi Zhang ${ }^{1}$, Yi-Lan Shen ${ }^{1}$, Wen-Long Feng ${ }^{2}$ and Zhi-Gang Wang ${ }^{2}$ \\ ${ }^{1}$ Grade 14 Mathematics and Applied Mathematics, School of Information Science and \\ Technology, Hainan University, Haikou, 570228, China \\ 2School of Information Science and Technology, Hainan University, Haikou, 570228, \\ China \\ E-mail:1400517163@qq.com and1092923790@qq.com,wzhigang@hainu.edu.cn
}

\begin{abstract}
The emphasis on this paper is mainly on the empirical study of market share and marketing strategy based on Markov chain model. Firstly, we review basic theory of Markov chain model. Secondly, two examples are cited to analyze the market share and marketing strategy, and MATLAB 7.0 is used to calculate the numerical solution. By scientific and reasonable information of the market share and marketing strategy, we try to help enterprises achieve the objective of the maximum expected profit.

Keywords: Markov Chain Model; Transition Probability Matrix; Market Share; Marketing Strategy; The Expected Profit.
\end{abstract}

\section{Guidelines}

Markov chain model is widely used in the field of automatic control, communication technology, biological engineering, economic management and so on. Markov chain model in random environments is developing rapidly and has been a very important research area in the stochastic processes. It is necessary requirement that leads the theoretical research about stochastic processes to develop in depth and width, and it also has very important theoretical meaning and broad applied prospects. Su [1]studied the identification and measurement of contagion effects of the crises Based on improved Markov independent switching dynamic conditional correlation model analysis.Hu[2] considered application of Markov china in domain name system information detection, and so on.

The emphasis on this paper is mainly on the empirical study of market share and marketing strategy based on Markov chain model. The rest of the paper is organized as follows. Firstly, we review basic theory of Markov chain. Secondly, two examples are cited to analyze the market share and marketing strategy, and 
the use of MATLAB 7.0 to calculate the numerical solution. By scientific and reasonable information of market occupation rate and promotion strategy, we try to help enterprises achieve the objective of the maximum expected profit according to market analysis and different selling states.

\section{Review on Markov Chain Model}

Markov chain model is a probability forecast method and technique which forecast the next states of the system by studying current state and its change tendency of the system.

Let $\left\{X_{n}, n \in T\right\}$ be stochastic processes, the state set of the categorical data sequence be $I$. for all integer $n \in T$ and $i_{0}, i_{1}, \cdots, i_{n} \in I$, the conditional probability satisfy the following equation

$$
P\left\{X_{n+1}=i_{n+1} \mid X_{0}=i_{0}, X_{1}=i_{1}, \ldots X_{n}=i_{n}\right\}=P\left\{X_{n+1}=i_{n+1} \mid X_{n}=i_{n}\right\}
$$

then $\left\{X_{n}, n \in T\right\}$ is called Markov chain. The intuitive explanation of Markov chain property can be expressed as follows: the "past" and the "future" are conditionally independent given the "present". The conditional probability

$$
p_{i j}(n)=P\left\{X_{n+1}=j \mid X_{n}=i\right\} \text { is called one-step transition probability, its intuitive }
$$
means is that the system is in the state " $i$ " at time " $n$ ", while being the state " $j$ " at time " $n+1$ ".that means the system transit from one state to another state through only unit time. We obtain the following transition probability matrix

$$
P=\left(p_{i j}\right)_{n \times n}
$$

Where $p_{i j}$ is the conditional probability from state $i$ to state $j$ which satisfy the following conditions $0 \leq p_{i j} \leq 1$ and $\sum_{i \in I} p_{i j}=1$. The k-step of Markov model lies in getting primary distribution and transition probability . If we acquire primary distribution vector $P^{T}(0)=\left(p_{0}, p_{1}, \cdots\right)$ and matrix (1), we obtain its absolutely distribution $P^{T}(n)=\left\{p_{1}(n), p_{2}(n), \cdots\right\}=P^{T}(0) P$.

There exists one distribution $\left\{\pi_{j}, j \in I\right\}$, such that $\pi_{j}=\sum_{i \in I} \pi_{i} p_{i j}$ and $\sum_{i \in I} \pi_{i}=1$, then $\left\{\pi_{j}, j \in I\right\}$ is called its stationary distribution. Suppose that Markov china which primary distribution $P^{T}(0)$ is stationary distribution, then for all positive integer $n$, it's absolute distribution $P^{T}(n)$ is equal to its primary distribution, which is the stationary distribution. If non- periodic irreducible Markov chain is positive recurrent, then there exists a stationary distribution which is its limit distribution. 


\section{Two Empirical Analysis}

\subsection{Example 1 Market Share Forecast}

Suppose that a certain product sell's market has $A, B, C 3$ kinds of products. The following Tab. 1 and Tab. 2 provide the market share at time of 0,1,2, and the transition probability matrix, respectively. Firstly, assumed states 1,2,3 represent the product $A, B, C$, respectively. $\left\{X_{n}\right\}$ to be production choice at the time of $n$. By inspecting two transition probability matrix $P_{1}$ and $P_{2}$, we cannot reject the presumation that the transition probability exists stationary distribution. Utiliting the second maket occdupation rate $S^{(2)}$ and the transition paobability matr $P_{2}$, we predict the next time maket occdupation rate $S^{(3)}=S^{(2)} P_{2}$. That is $S^{(3)}=(0.234,0.483,0.283)$

Tab. 1 Sampled data of market share

\begin{tabular}{lccccl}
\hline Market share & A & B & C & Total \\
\hline $\mathrm{S}(0)$ & 170 & 520 & 310 & 1000 \\
& 0.17 & 0.52 & 0.31 & 1 \\
$\mathrm{~S}(1)$ & 200 & 500 & 300 & 1000 \\
& 0.2 & 0.5 & 0.3 & 1 \\
$\mathrm{~S}(2)$ & 220 & 490 & 290 & 1000 \\
& 0.22 & 0.49 & 0.29 & 1 \\
\hline
\end{tabular}

Tab. 2. Transition Probability Matrix

\begin{tabular}{|c|c|c|}
\hline A & $\mathrm{B} \mathrm{C}$ & A B \\
\hline $\mathrm{P} 1: 0 \rightarrow 1 \mathrm{~A}$ & $\begin{array}{llll}0.70 & 0.17 & 0.13\end{array}$ & P2: $1 \rightarrow 0 \quad A \quad 0.80 \quad 0.10 \quad 0.10$ \\
\hline B 0.05 & 0.910 .04 & B $\quad 0.07 \quad 0.90 \quad 0.03$ \\
\hline C 0.17 & 0.130 .70 & $\begin{array}{llll}\mathrm{C} & 0.083 & 0.067 & 0.85\end{array}$ \\
\hline
\end{tabular}

If we choose $P_{2}$ to be stationary transition probability matrix,. By the Markov chain theory, It is easy to obtain that $\left\{X_{n}, n \geq 0\right\}$ is irreducible ergodic Markov chain which exists stationary distribution which the stationary distribution is its limit distribution.

Let primary distribution $p_{i}=P\left\{X_{0}=i\right\}=\pi_{i}(i=1,2,3)$ be stationary distribution,its transition pribability matrix be 


$$
P=\left(\begin{array}{ccc}
0.8 & 0.1 & 0.1 \\
0.07 & 0.9 & 0.03 \\
0.083 & 0.067 & 0.85
\end{array}\right)
$$

By the stationary equation $\pi_{j}=\sum_{i \in I} \pi_{i} p_{i j}$ and $\sum_{i \in I} \pi_{i}=1$, the limit distribution is obtained by MATLAB $\pi_{j}=(0.273,0.454,0.273)$

It is to say that the finite market occupation rate of the products $A B C$ is $27.3 \%, 45.4 \%, 27.3 \%$, respectively, on the condition of the stationary market environments.

\subsection{Example 2 Marketing Strategy of Product Sales Market}

A certain refrigerator enterprise try to achieve the objective of the maximum expected profit according to different sales state. Suppose that sales state of enterprise are divided into selling and unsalable state. In each sales state. The enterprise adopt to corresponding strategy by depreciating sales promotion (strategy 1)and increasing advertising devotion(strategy 2), respectively. By marketing survey chronically, we establish the expected profit table (Tab. 3).

Tab. 3 The expected profit at time

\begin{tabular}{|c|c|c|c|c|c|c|}
\hline States & Strategy k & \multicolumn{4}{|c|}{$\begin{array}{l}\text { Transition probability Profit } \\
\text { Kpi1 kpi2 kri1 kri2 }\end{array}$} & $\begin{array}{c}\text { Expect } \\
\text { ed } \\
\text { profit }\end{array}$ \\
\hline A & 1 & 0.8 & 0.2 & 1.6 & 1.0 & 1.48 \\
\hline \multicolumn{2}{|r|}{2} & 0.9 & 0.1 & 1.8 & 0.8 & 1.70 \\
\hline B & 1 & 0.6 & 0.4 & 0.6 & -1.0 & -0.04 \\
\hline \multicolumn{2}{|r|}{2} & 0.7 & 0.3 & 0.8 & -2.1 & -0.07 \\
\hline
\end{tabular}

Note: A represents the selling state; $\mathrm{B}$ represents the unsalable state; 1 represents the price promotion strategy;2represents the advertising promotion strategy.

Let $S_{i}(m)(m=1,2, \cdots, n)$ be the strategy on the state $i(i=1,2)$ at time $m ; M_{k}(n)$ be the maximum expected profit undergo $n$ units time on the state $i . q_{i}(k)(i=1,2)$ be the expected profit in time through taking strategy $k$ at time $i$. The following equation is trivially.

$$
q_{i}(k)=\sum_{j=1}^{2} k p_{i j} \cdot k r_{i j}
$$


$M_{i}(n)=\max _{1 \leq k \leq 2}\left\{\sum_{j=1}^{2} k p_{i j}\left[k p_{i j}+M_{j}(n-1)\right]\right\}=\max _{1 \leq k \leq 2}\left\{q_{i}(k)+\sum_{j=1}^{2} k p_{i j} \cdot M_{j}(n-1)\right\}$

By the Eq. (3), we obtain

$$
\begin{aligned}
& M_{1}(1)=\max \left\{\sum_{j=1}^{2} 1 p_{1 j} \cdot 1 r_{1 j}, \sum_{j=1}^{2} 2 p_{1 j} \cdot 2 r_{1 j}\right\} \\
& =\max \{0.8 \times 1.6+0.2 \times 1.0,0.9 \times 1.8+0.1 \times 0.8\}=\max \{1.48,1.70\}=1.70
\end{aligned}
$$

That is, $S_{1}(1)=2$

$$
\begin{aligned}
& M_{2}(1)=\max \left\{\sum_{j=1}^{2} 1 p_{2 j} \cdot 1 r_{2 j}, \sum_{j=1}^{2} 2 p_{2 j} \cdot 2 r_{2 j}\right\} \\
& =\max \{0.6 \times 0.6-0.4 \times 0.1,0.7 \times 0.8-0.8 \times 2.1\} \\
& =\max \{-0.04,-0.07\}=-0.04 \\
& S_{2}(1)=1 \\
& M_{1}(2)=\max \{1.48+0.8 \times 1.70+0.2 \times(-0.04), \\
& 1.70+0.9 \times 1.70+0.1 \times(-0.04)\}=\max \{2.832,3.226\}=3.226 \\
& \quad S_{1}(2)=2 \\
& M_{2}(2)=\max \{-0.04+0.6 \times 1.70+0.4 \times(-0.04), \\
& =\max \{0.964,1.108\}=1.108, S_{2}(2)=2, \cdots \cdots
\end{aligned}
$$

In a similar way, the use of MATLAB to calculate the expected profit under the corresponding marketing strategy at time $n$ ( Tab. 4).Tab. 4 data show that, in the best-selling state, after 5 units of time, the use of advertising sales promotion strategy can achieve the maximum expected profit 7.572. Conversely, in the slow-moving state, after 4 units of time, price promotions can maximize the expected return of 4.375 .

Tab. 4 The maximum expected profit and corresponding strategy

\begin{tabular}{lllllllll}
\hline $\mathrm{N}$ & 1 & 2 & 3 & 4 & 5 & \\
\hline $\mathrm{M} 1(\mathrm{n})$ & 1.70 & 3.226 & 4.714 & 6.110 & 7.572 & ---- \\
$\mathrm{M} 2(\mathrm{n})$ & -0.04 & 1.108 & 1.673 & 3.732 & 4.375 & ---- \\
$\mathrm{S} 1(\mathrm{n})$ & 2 & 2 & 2 & 2 & 2 & & ---- \\
\hline
\end{tabular}

Markov chain model comparatively neatly reflect to the development diversification of the promotion activity. The conclusion is objective and credible. Since transition probability matrix contains a great lot of information, we convenient for the diversification state that of market, it is propitious to forecast sales state chronically and adopt corresponding promotion strategy . 


\section{Acknowledgments}

This work is supported by the National Science and Technology Support Program (Grant No. 2015BAH55F01), the Natural Science Foundation of Hainan Province (Grant No.20161002) and the Education and Teaching Reform Research Project of Hainan University ( Grant No. hdjy1639).

\section{References}

1. Su H J, Ouyang H B, Identification and measurement of contagion effects of the crises based on improved Markov independent switching dynamic conditional correlation model analysis[J].Journal of Management Sciences in China. 16 (8), 2013: 20-30.

2. Hu R G, Xu C X, Wang Y Y, Zhang L. Application of Markov china in domain name system information detection[J]. Computer Applications and Software. 2015,32(6):152-155.

3. Sun R H, Stochastic processes and its applications [M], Tsinghua University Publishing Company, Beijin, 2005.

4. Liu Y Y, Hou Z T, Exponential and strong ergodicity for Markov processes with an application to queues, Chinese annals of mathematics ,Series B, 2008,29(B):199-202.

5. Wang Z G, Ou Y G. Markov chain evaluation of teaching quality[J]. Proceeding of the seventh International Conference on Information and Management Sciences, Vol,7,2008:286-288.

6. Wang C, Huang T Z. A new higher-order multivariate Markov chain model[J]. Chinese Journal of Engineering Mathematics. 2015, 32(3):462474.

7. Ding Y C, Zhu H, Zhong S M. H filtering for stochastic systems with Markov switching and partly unknown transition probabilities[J]. Circuits Systems and Signal Process, 2013, 32(2): 559-583.

8. Li J N, Pan Y J, Su H Y, et al. Stochastic reliable control of a class of networked control systems with actuator faults and input saturation[J]. Int $\mathbf{J}$ of Control, Automation and Systems, 2014, 12(3): 564-571.

9. Liu Y Q, Liu F. Disturbance rejection for Markov jump systems with partly unknown transition probabilities and saturation[J]. Circuits Systems and Signal Processing, 2013, 32(6): 2783-2797.

10. Zhao J J, Wang J, Park J H, et al. Memory feedback controller design for stochastic Markov jump distributeddelay systems with input saturation and partially knowntransition rates[J]. Nonlinear Analysis: Hybrid Systems, 2015, 15: 52-62. 